\title{
Tardigrades Research in Brazil: an overview and updated checklist
}

\author{
Rodolfo Corrêa de Barros' \\ 1 Universidade Positivo (UP), Ciências Biológicas (CBI0). Curitiba, PR, Brasil. \\ ORCID: http://orcid.org/0000-0003-0801-9649. E-mail: rodolfo.barros@up.edu.br
}

\begin{abstract}
Tardigrades are microscopic animals, commonly referred to as "water bears", and comprise the phylum Tardigrada. They are found in diverse habitats in terrestrial, freshwater and marine environments worldwide. In this paper, it is presented a brief history of the study of tardigrades in Brazil and an updated species checklist of Brazilian tardigrades. Since the first report in 1913, the number of tardigrades records has increased, reflecting advances in the understanding the diversity of tardigrades in Brazil. A total of 100 species known from Brazil are listed, being 30 in marine and 70 in terrestrial and freshwater environments. The records are concentrated in Southeast (47.1\%) and Northeast (41.3\%) regions. Despite the advances, further research and sampling of new areas is still needed, besides reanalysis and confirmation of old records. Brazil, with its vast territory, extensive continental shelf and great diversity of biomes, has great potential to expand our knowledge of tardigrades fauna.
\end{abstract}

Key-Words. Eutardigrada; Heterotardigrada; Tardigrada; Exploration history.

\begin{abstract}
Resumo. Tardígrados, também conhecidos como "ursos d'água", são animais microscópicos que compõem o filo Tardigrada. São encontrados em diversos habitats de ambientes terrestres, de água doce e marinha. Uma breve história do estudo dos tardígrados no Brasil é apresentada, bem como a lista atualizada de espécies de tardígrados brasileiros. Desde o primeiro relato em 1913, o número de registros de tardígrados aumentou, refletindo os avanços no entendimento da diversidade brasileira de tardígrados. São listadas 100 espécies conhecidas para 0 Brasil, sendo 30 em ambientes marinhos e 70 em ambientes terrestres e de água doce. Os registros estão concentrados nas regiões Sudeste (47,1\%) e Nordeste (41,3\%). Apesar dos avanços, ainda são necessárias mais pesquisas e amostragens de novas áreas, além da reanálise e confirmação dos registros antigos. 0 Brasil, com seu vasto território, extensa plataforma continental e grande diversidade de biomas, tem um grande potencial para expandir nosso conhecimento sobre a fauna de tardígrados.
\end{abstract}

Palavras-Chave. Eutardigrada; Heterotardigrada; Tardigrada; História de exploração.

\section{INTRODUCTION}

Tardigrades are microscopic Ecdysozoans (Aguinaldo et al., 1997), with $0.5 \mathrm{~mm}$ to $1.2 \mathrm{~mm}$ in length (excluding the last pair of legs), four pairs of locomotor appendages (lobopodous), generally ending with claws of varying numbers and shapes (Ramazzotti \& Maucci, 1983). These animals are commonly referred to as "water bears" due to their bear-like appearance (legs with claws), and slow lumbering gait (Nelson et al., 2015).

Since the first observation in 1773, 1,298 species of tardigrades (142 genera and 30 families) have been described, including two fossils species (Degma et al., 2019). Two classes and four orders were accepted in the Tardigrada phylum. Eutardigrada constituted by Apochela and Parachela, and Heterotardigrada constituted by
Echiniscoidea and Arthrotardigrada (Jørgensen et al., 2018). A third class, Mesotardigrada, was established by Rahm in 1937 based on the description of Thermozodium esakii Rahm, 1937 from a Japanese hot spring (Nelson et al., 2015). However, the type material was lost, so Mesotardigrada is considered nomen dubium until new evidence is found (Grothman et al., 2017). Recently, based on molecular data, Guil et al. (2019) proposed the creation of a new class, Apotardigrada, constituted by order Apochela, and consequently suppression of order Parachela and its superfamilies erected as orders (Fig. 1).

The tardigrades occupy a variety of habitats; they can be found in marine, freshwater, and terrestrial environments worldwide (Nelson, 2002). Heterotardigrada, with few exceptions, encompasses marine tardigrades that inhabit the inter- 
Apotardigrada (Guil et al., 2019) comb. $\mathrm{n}$ - Apochela Schuster et al., 1980

\begin{tabular}{l|l} 
Macrobiotoidea Thulin 1928 comb. n \\
Isohypsibioidea Sands et al., 2008 com
\end{tabular} Heterotardigrada Marcus, $1927 \mid \begin{aligned} & \text { Arthrotardigrada Marcus, } 1927 \\
& \text { Echiniscoidea Richters, } 1926\end{aligned}$

Eohypsibioidea Bertolani \& Kristensen, 1987 comb. $n$.

\begin{tabular}{|l|l} 
Eutardigrada Marcus, 1927 & $\begin{array}{l}\text { Hypsibioidea Pilato, } 1969 \text { comb. } \mathrm{n} . \\
\text { Macrobiotoidea Thulin } 1928 \text { comb. } \mathrm{n} . \\
\text { Isohypsibioidea Sands et al., } 2008 \mathrm{comb} . \mathrm{n} .\end{array}$
\end{tabular}

Echiniscoidea Richters, 1926

Figure 1. Systematics of Tardigrada according to proposal of Guil et al., 2019.

tidal zone and shallow waters of the continental shelf as well as the deep-sea benthic sediments. On the other hand, Eutardigrada, and the new class Apotardigrada, mainly contain both terrestrial and freshwater species (Nelson et al., 2015; Guil et al., 2019).

In Brazil, however, tardigrades are still poorly known; there are about 80 taxa recorded in eight Brazilian states: Ceará, Pernambuco, Rio Grande do Norte, Minas Gerais, São Paulo, Rio de Janeiro, Rio Grande do Sul and Paraná (Gomes Júnior, 2015). The little information about the tardigrades fauna and their distribution in Brazilian regions are mainly a result of a scarcity of specialists and of taxonomic studies about the group. It is necessary to promote further studies and stimulate younger biologists to dedicate themselves to the study of the tardigrades in Brazil. To start this, it seems useful to remember the efforts made in the past and to know the current state-ofthe-art, including the species already known. Thus, this paper presents a brief history of the study of tardigrades in Brazil and provides an updated species checklist of Brazilian tardigrades.

\section{On tardigrades research In Brazil}

The first work to report a tardigrade species in Brazil dates back to the early twentieth century, when the Scottish naturalist James Murray recorded the occurrence of Macrobiotus occidentalis Murray, 1910; however, without specifying the Brazilian location (Murray, 1913). It was almost two decades after that new records of tardigrades were made. In 1931, the German zoologist Gilbert (Franz) Rahm reported the occurrence of the limnoterrestrial (non-marine) species Echiniscus blumi Richters, 1903, Echiniscus testudo trifilis (Doyère, 1840), Macrobiotus ambiguus Murray, 1907, Milnesium tardigradum trispinosum Doyère, 1840, Hypsibius alpinus Murray, 1906 and Hypsibius oberhauseri Doyère, 1840 in bromeliad and mosses from São Paulo; Echiniscus arctomys Ehrenberg, 1853 in mosses from Rio de Janeiro;
Pseudechiniscus bispinosus Murray, 1907 in mosses from Pernambuco; Macrobiotus echinogenitus Richters, 1903 and Macrobiotus hufelandi Schultze, 1834 in mosses from Pernambuco and São Paulo; Milnesium tardigradum Doyère, 1840 in mosses from Pernambuco, Rio de Janeiro and São Paulo (Rahm, 1931). In the next year, Rahm added Pseudechiniscus suillus papillata Ehrenberg, 1853 (mosses from Rio de Janeiro) in the previous list and dated the record of Milnesium tardigradum in Pernambuco state from 1928 (Rahm, 1932).

The German naturalist couple Ernest Gustav Gotthelf Marcus and Eveline du Bois-Reymond Marcus were the first researchers to study tardigrades in a Brazilian institution. In 1936, Marcus and his wife left Berlin to live in São Paulo, as Ernest Marcus had accepted an invitation to head the Zoology Department at the University of São Paulo, from 1936 to 1963 (Corrêa, 1991; Edmunds, 1991; USP, 2019). Eveline and Ernest Marcus received Brazilian citizenships in 1940 and never again left the country (Corrêa, 1991). Together, Eveline and Ernest Marcus published over 280 papers on varied taxonomic groups (Sawaya, 1970; Corrêa, 1991), 27 of them on anatomy, histology, physiology, embryology, ecology, and systematics of tardigrades. Some articles published in São Paulo were written in Portuguese, as a way of honoring Brazil (Mendes, 1994); these included important works as "Sôbre a anabiose dos Tardigrados, com descrição duma nova especie" (Marcus, 1937), and "Sobre Tardígrados Brasileiros" (du Bois-Reymond Marcus, 1944). Eveline and Ernest Marcus described several new species of tardigrades, such as Macrobiotus sawayai Marcus, 1937 (Marcus, 1937), Batillipes pennaki Marcus, 1946 (Marcus, 1946), and Orzeliscus belopus du Bois-Reymond Marcus, 1952 (du Bois-Reymond Marcus, 1952); they were the pioneers in the study of marine tardigrades on the Brazilian coast.

At the same time as Eveline and Ernest Marcus, Rosina de Barros was the first Brazilian researcher to dedicate herself to the study of tardigrades. Before joining geneticist Andre Dreyfus's team in 1943 (Formiga, 2010), 
Barros had already published important works on the taxonomy of tardigrades, paying special attention to the state of São Paulo. Between 1938 and 1943, Barros released substantial papers (see Barros 1938, 1939a, b, $1942 a, b, 1943)$, providing a profound basis for subsequent studies. The papers of Barros are the milestone in the Brazilian tardigradology. Barros described one genus, eight species, and four subspecies (Assunção, 1999b), including Macrobiotus evelinae Barros, 1938 (Barros, 1938), Macrobiotus hibiscus Barros, 1942 (Barros, 1942b), Macrobiotus primitivae Barros, 1942 (Barros, 1942b), Itaquascon umbellinae Barros, 1939 (Barros, 1939a), and Pseudobiotus juanitae Barros, 1939 (Barros, 1939b). The works of Ernest and Eveline Marcus, and Rosina de Barros represent the birth of Brazilian tardigradology.

Samples collected from Manaus, in the Amazon region, in September 1966 contained Paramacrobiotus richtersi (Murray, 1911) (Iharos, 1969). Höfling-Epiphanio (1972) registered Batillipes mirus Richters, 1909, and Batillipes tubernatis Pollock, 1971 on the São Paulo coast. Renaud-Mornant described one new species, Tanarctus heterodactylus Renaud-Mornant, 1980, and two new genera, Chrysoarctus Renaud-Mornant, 1984, and Opydorscus Renaud-Mornant, 1989, from the continental shelf of Brazil (Renaud-Mornant 1980, 1984, 1989). Two new species were described from the Iguazu falls (state of Paraná), Isohypsibius sabellai Pilato, Binda, Napolitano \& Moncada, 2004 (Pilato et al., 2004), and Paramacrobiotus centesimus (Pilato, 2000) (Pilato, 2000). Other contributions were made from different laboratory techniques, including the collection and fixation of marine and freshwater tardigrades (Corrêa, 1987), laboratory culture methods of limnoterrestrial tardigrades (Pulschen \& Meneghin, 2010) and protocols for processing tardigrades samples for scanning electron microscopy analysis (Gomes Júnior \& Rocha, 2015).

Using morphological data, the biologist Cláudia Maria Leite Assunção was the first Brazilian researcher to explore the tardigrades phylogeny. In her PhD thesis, Assunção investigated the relationship between the subgroups Stygarctidae and Digitopoda, resulting in the establishment of Stygarctidae as a monophyletic taxon, and Digitopoda was branched into two clades (Assunção, 1994, 2001). The biologist Clélia Márcia Cavalcanti da Rocha maintains a research group on the systematics and ecology of tardigrades, working mainly with marine tardigrades of the northeastern coast of Brazil (Rocha, 2018). Recently, her group described the new species Ligiarctus alatus Gomes Júnior, Santos, da Rocha, Santos \& Fontoura, 2018 (Gomes Júnior et al., 2018), and three new Batillipes species from the Brazilian coast: Batillipes dandarae Santos, Rocha, Gomes Jr. \& Fontoura, 2017; Batillipes potiguarensis Santos, Rocha, Gomes Jr. \& Fontoura, 2017; and Batillipes brasiliensis Santos, Rocha, Gomes Jr. \& Fontoura, 2017 (Santos et al., 2017). Rocha also organized the first tardigrades scientific collection in Brazil (Rocha, 2018). Since 2017, the biologist André Rinaldo Senna Garraffoni has been developing a project that intends to redescribe all species with missing type material, whose type locality is in the state of São Paulo, thus increasing the number of nuclear (18S and 28S) and mitochondrial (COI) gene sequences of the Brazilian tardigrades species deposited in Genbank (Garraffoni, 2019).

Until the late twentieth century, the tardigrades were sporadically studied in Brazil. However, despite of historical gaps, the future of tardigrades research in Brazil seems promising. The presence of researchers interested on tardigrades in the different regions of Brazil (Northeast, Southeast and South), the training of new researchers (e.g., Edivaldo Lima Gomes Júnior, Érika Santos, Mônica Marinho Verçosa, and Paola Visnardi Fassina), the increasing of Brazilian tardigrades records and the recent description of new species from Brazilian specimens, are pointing to new horizons. On the other hand, to strengthening the Brazilian tardigradology, it would be interesting the establishment of a cooperation network among Brazilian tardigrades researchers, in order to ensure the exchange of information and expertise.

\section{On tardigrades diversity in Brazil}

There are four relevant species surveys of tardigrades in Brazil: Assunção (1999a, b) listed six marine and 58 limnoterrestrial species for the state of São Paulo; Rocha et al. (2013) listed 27 marine species for the Brazilian coast; Gomes Júnior (2015) listed 80 tardigrades species, both marine and limnoterrestrial, and Rocha et al. (2016) updated the Brazilian limnoterrestrial checklist to 62 taxa. In the present update, the number of tardigrades records in marine environment increased to 30 (Table 1) and to 70 in terrestrial and freshwater environments (Table 2). There are fewer studies on freshwater and marine species compared to terrestrial species, and the major samples investigated were sediment (mud, sand and gravel) to marine, and mosses to limnoterrestrial tardigrades. Compared to Gomes Júnior's (2015) checklist, it was not found any record on tardigrade species in the state of Rio Grande do Sul. On the other hand, it was found one record in the state of Amazonas and new records in the state of Alagoas. The records are concentrated in Southeast (47.1\%) and Northeast regions (41.3\%) and reflect the historical construction of the Brazilian tardigradology and, consequently, the distribution of researchers (Fig. 2).

This sampling effort represents minimal due to the extensive continental dimension of Brazil. According to Kaczmarek et al. (2015a) over $65 \%$ of the Brazilian terrestrial area is undocumented. However, if we take into account the geographic extension of the country (8.511 million $\mathrm{km}^{2}$ ), the territorial sea (3.6 million $\left.\mathrm{km}^{2}\right)$, the richness of habitats and variety of Brazilian biomes, the percentage should be much higher, perhaps over 99\%. In other words, the vast Brazilian territory virtually remains unexplored with respect to tardigrades.

While recognizing the advances in understanding biodiversity of Brazilian tardigrades in the last years, further research and sampling of new areas is still needed, particularly in those states not yet sampled. Old records, 
Table 1. Marine tardigrades recorded in Brazilian coast.

\begin{tabular}{lcl}
\hline \multicolumn{1}{c}{ Taxon } & State & \multicolumn{1}{c}{ Substratum } \\
\hline Actinarctus doryphorus doryphorus & $\mathrm{CE}, \mathrm{RN}$ & gravel, sand \\
Archechiniscus marci & $\mathrm{CE}, \mathrm{RN}$ & sand, mud \\
Angursa lingua & $\mathrm{CE}, \mathrm{RN}$ & sand, mud \\
Batillipes annulatus & $\mathrm{CE}, \mathrm{PE}$ & sand \\
Batillipes brasiliensis & $\mathrm{AL}$ & gravel, sand \\
Batillipes dandarae & $\mathrm{AL}$ & sand \\
Batillipes dicrocercus & $\mathrm{CE}, \mathrm{PE}$ & sand \\
Batillipes lesteri & $\mathrm{CE}, \mathrm{PE}, \mathrm{RN}$ & sand \\
Batillipes mirus & $\mathrm{SP}$ & sand \\
Batillipes pennaki & $\mathrm{AL}, \mathrm{CE}, \mathrm{PE}, \mathrm{RJ}, \mathrm{SP}$ & sand, mud, sea water \\
Batillipes potiguarensis & $\mathrm{RJ}, \mathrm{RN}$ & gravel, sand \\
Chrysoarctus briandi & $\mathrm{CE}, \mathrm{RJ}, \mathrm{RN}$ & sand, mud \\
Dipodarctus subterraneus & $\mathrm{PE}$ & macroalgae (Halimeda opuntia) \\
Echiniscoides sigismundi sigismundi & $\mathrm{SP}$ & barnacles \\
Florarctus hulingsi & $\mathrm{CE}, \mathrm{RN}$ & gravel, sand \\
\hline
\end{tabular}

\begin{tabular}{lcl}
\hline \multicolumn{1}{c}{ Taxon } & State & \multicolumn{1}{c}{ Substratum } \\
\hline Halechiniscus perfectus & $\mathrm{PE}, \mathrm{RN}$ & sand \\
Halechiniscus tuleari & $\mathrm{PE}$ & gravel \\
Ligiarctus alatus & $\mathrm{RN}$ & sand \\
Mesostygarctus intermedius & $\mathrm{CE}, \mathrm{PE}, \mathrm{RN}$ & sand, mud \\
Neoarctus sp. & $\mathrm{CE}, \mathrm{RN}$ & mud \\
Neostygarctus sp. & $\mathrm{CE}, \mathrm{RN}$ & mud \\
Opydorscus fonsecae & $\mathrm{CE}, \mathrm{PE}, \mathrm{RN}$ & sand, mud \\
Orzeliscus belopus & $\mathrm{AL}, \mathrm{CE}, \mathrm{PE}, \mathrm{RN}, \mathrm{SP}$ & sand, mud \\
Parastygarctus sterreri & $\mathrm{CE}, \mathrm{PE}, \mathrm{RN}$ & sand, mud \\
Raiarctus aureolatus & $\mathrm{CE}, \mathrm{RN}$ & sand, mud \\
Stygarctus bradypus & $\mathrm{PE}$ & sand \\
Tanarctus dendriticus & $\mathrm{CE}, \mathrm{RN}$ & mud \\
Tanarctus heterodactylus & $\mathrm{CE}, \mathrm{PE}, \mathrm{RJ}, \mathrm{RN}$ & gravel, sand, mud \\
Tanarctus velatus & $\mathrm{CE}, \mathrm{RN}$ & sand, mud \\
Wingstrandarctus intermedius & $\mathrm{CE}, \mathrm{PE}$ & macroalgae (Halimeda opuntia) \\
\hline
\end{tabular}

$\mathrm{AL}=$ Alagoas; $\mathrm{CE}=$ Ceará; $\mathrm{PE}=$ Pernambuco; $\mathrm{RJ}=$ Rio de Janeiro; $\mathrm{RN}=$ Rio Grande do Norte; $\mathrm{SP}=$ São Paulo.

Table 2. Limnoterrestrial tardigrades recorded in Brazil.

\begin{tabular}{|c|c|c|}
\hline Species & State & Substratum \\
\hline Adropion scoticum scoticum & SP & mosses, aquatic plants \\
\hline Bryochoerus intermedius intermedius & SP & mosses \\
\hline Bryodelphax alzirae & SP & mosses, aquatic plants \\
\hline Bryodelphax parvulus & SP & mosses \\
\hline Dactylobiotus ambiguus & SP & bromeliads \\
\hline Dianea papillifera & SP & mosses, aquatic plants \\
\hline Diphascon alpinum & SP & mosses \\
\hline Diphascon pingue pingue & SP & mosses, aquatic plants \\
\hline Doryphoribius evelinae & $M G, R J, S P$ & mosses, aquatic plants \\
\hline Doryphoribius flavus & PE & mosses \\
\hline Echiniscus arctomys & RJ & mosses, algae \\
\hline Echiniscus blumi blumi & SP & mosses \\
\hline Echiniscus crassispinosus crassispinosus & PR, SP & mosses, aquatic plants \\
\hline Echiniscus crassispinosus fasciatus & $\mathrm{PR}, \mathrm{SP}$ & mosses, aquatic plants \\
\hline Echiniscus dreyfusi & SP & mosses \\
\hline Echiniscus duboisi & SP & mosses \\
\hline Echiniscus evelinae & PR, SP & mosses, aquatic plants \\
\hline Echiniscus spiniger & SP & mosses, aquatic plants \\
\hline Echiniscus tenuis & SP & mosses, aquatic plants \\
\hline Echiniscus testudo & $S P$ & mosses \\
\hline Fractonotus verrucosus & SP & mosses, aquatic plants \\
\hline Grevenius granulifer & $S P$ & unknown \\
\hline Grevenius myrops & $\mathrm{PR}, \mathrm{SP}$ & aquatic plants \\
\hline Hypsibius convergens & SP & mosses, aquatic plants \\
\hline Hypsibius microps & $S P$ & unknown \\
\hline Isohypsibius sabellai & PR & mosses \\
\hline Itaquascon umbellinae & SP & mosses \\
\hline Macrobiotus echinogenitus & $\mathrm{PE}, \mathrm{SP}$ & mosses \\
\hline Macrobiotus evelinae & $S P$ & mosses \\
\hline Macrobiotus furcatus & SP & mosses, aquatic plants \\
\hline Macrobiotus hibiscus & SP & unknown \\
\hline Macrobiotus hufelandi & $\mathrm{PE}, \mathrm{SP}$ & mosses, aquatic plants \\
\hline Macrobiotus occidentalis occidentalis & PR & unknown \\
\hline Macrobiotus polyopus & SP & unknown \\
\hline Macrobiotus primitivae & $\mathrm{PR}, \mathrm{SP}$ & mosses, aquatic plants \\
\hline
\end{tabular}

\begin{tabular}{|c|c|c|}
\hline Species & State & Substratum \\
\hline Macrobiotus psephus & $S P$ & unknown \\
\hline Macrobiotus sawayai & SP & mosses \\
\hline Mesobiotus coronatus & SP & unknown \\
\hline Mesobiotus furciger & SP & unknown \\
\hline Mesobiotus harmsworthi & $P E, P R, S P$ & mosses, aquatic plants \\
\hline Mesobiotus orcadensis & SP & unknown \\
\hline Mesobiotus stellaris & SP & unknown \\
\hline Milnesium tardigradum tardigradum & \multicolumn{2}{|c|}{ PE, PR, RJ, SP mosses, algae } \\
\hline Milnesium tardigradum trispinosa & SP & mosses \\
\hline Minibiotus acontistus & SP & unknown \\
\hline Minibiotus aculeatus & PE & mosses \\
\hline Minibiotus intermedius & $P R, S P$ & mosses, aquatic plants \\
\hline Minibiotus julietae & SP & unknown \\
\hline Minibiotus marcusi & SP & unknown \\
\hline Mopsechiniscus imberbis & SP & unknown \\
\hline Mopsechiniscus schusteri & Unknown & unknown \\
\hline Murrayon pullari & SP & aquatic plants \\
\hline Nebularmis phocae & SP & mosses, aquatic plants \\
\hline Paramacrobiotus (Amicrobiotus) cf. areolatus & PR & mosses \\
\hline Paramacrobiotus (Amicrobiotus) centesimus & PR & mosses \\
\hline Paramacrobiotus (Paramacrobiotus) richtersi & AM, PR, SP & $\begin{array}{l}\text { leaf litter, mosses, aquatic } \\
\text { plants, bromeliads }\end{array}$ \\
\hline Pseudobiotus megalonyx & SP & mosses, aquatic plants \\
\hline Pseudechiniscus bispinosus & PE & mosses \\
\hline Pseudechiniscus facettalis & Unknown & unknown \\
\hline Pseudechiniscus juanitae & SP & mosses \\
\hline Pseudechiniscus novaezeelandiae novaezeelandiae & $P R, S P$ & mosses, aquatic plants \\
\hline Pseudechiniscus novaezeelandiae aspinosa & $P E, P R, S P$ & unknown \\
\hline Pseudechiniscus novaezeelandiae marinae & SP & mosses, aquatic plants \\
\hline Pseudechiniscus suillus & $P R, R J, S P$ & mosses, algae, aquatic plants \\
\hline Ramazzottius oberhaeuseri & $\mathrm{RJ}, \mathrm{SP}$ & mosses, aquatic plants \\
\hline Testechiniscus macronyx & SP & mosses \\
\hline Thulinius augusti & SP & unknown \\
\hline Ursulinius nodosus & SP & mosses, aquatic plants \\
\hline Viridiscus rufoviridis & SP & mosses, aquatic plants \\
\hline Viridiscus viridis & $\mathrm{PR}, \mathrm{SP}$ & mosses, lichens \\
\hline
\end{tabular}

$\mathrm{AM}=$ Amazonas; $\mathrm{MG}=$ Minas Gerais; $\mathrm{PE}=$ Pernambuco; $\mathrm{PR}=$ Paraná $; \mathrm{RJ}=$ Rio de Janeiro; $\mathrm{SP}=$ São Paulo. 


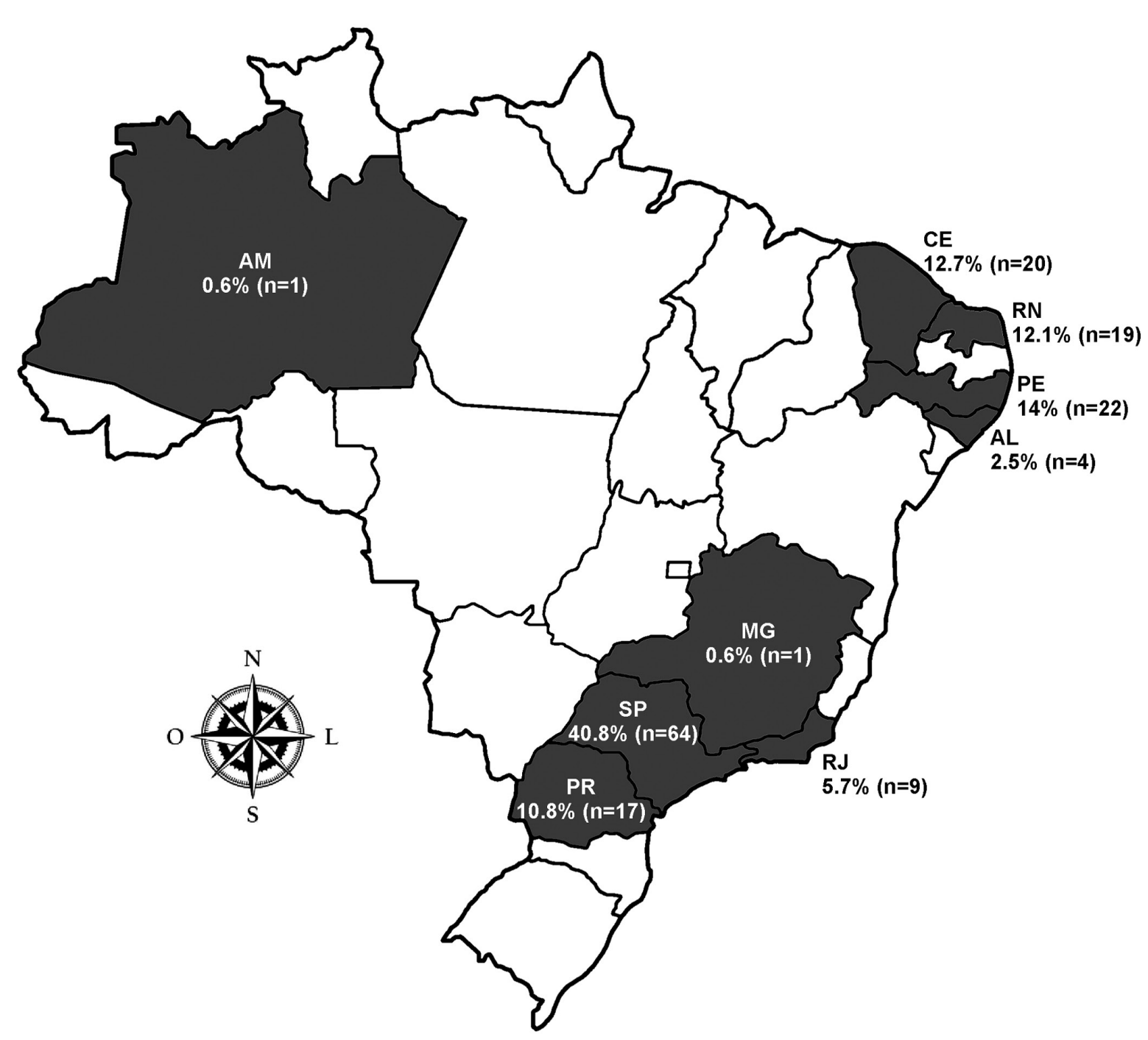

Figure 2. Brazilian records of tardigrades according the distribution frequency.

especially those that appeared only once, need to be confirmed with new specimens. Brazil, with its vast territory, extensive continental shelf and great diversity of biomes, has great potential to expand our knowledge of both marine and limnoterrestrial tardigrades fauna. Twenty-six tardigrade species (8 marine, 18 limnoterrestrial) have so far been originally described in Brazil, exhibiting this potentiality.

\section{MATERIAL AND METHODS}

Tardigrade species found in Brazil, according to the most recent classification (Degma et al., 2019), are shown in the following checklist. References are reported within parentheses '( )'. The species written in bold characters have Brazil as their type-locality. One species was invalidated, Echiniscus fischeri Richters, 1911 (Rocha et al., 2016), and 24 species were reallocated (for details, see species checklist). According to Santos et al. (2018b), the specimens from Brazil were wrongly identified for the species Batillipes tubernatis, so the previous records from the Pernambuco coast (Rocha et al., 2009) and São Paulo coast (Höfling-Epiphanio, 1972) do not belong to this species. For this reason, Batillipes tubernatis was left out of this Brazilian tardigrades checklist. On the other hand, although Macrobiotus sawayai Marcus, 1937 has been omitted from modern taxonomic literature and requires redescription (Kaczmarek et al., 2015a), it was kept on the checklist once its distribution is restricted to Brazil. Two new records from state of Paraná were included by the author: Paramacrobiotus (Amicrobiotus) cf. areolatus (Murray, 1907) sampled in mosses from Curitiba, and Viridiscus viridis (Murray, 1910) in lichens from Vila Velha state Park, Ponta Grossa (unpublished).

\section{RESULTS}

Phylum Tardigrada Doyère, 1840

Class Apotardigrada Guil, Jørgensen \& Kristensen, 2019 Order Apochela Schuster, Nelson, Grigarick \& Christenberry, 1980 Family Milnesiidae Ramazzotti, 1962

Milnesium tardigradum tardigradum Doyère, 1840 (Rahm, 1931, 1932; Marcus, 1936, 1939; Barros, 1943; du BoisReymond Marcus, 1944; Mclnnes, 1994; Assunção, 
1999b; Pulschen \& Meneghin, 2010; Meyer, 2013; Kaczmarek et al., 2015a; Rocha et al., 2016).

Milnesium tardigradum trispinosa Rahm, 1931 (Rahm, 1931, 1932; Marcus, 1939; Ramazzotti \& Maucci, 1983; Mclnnes, 1994; Kaczmarek et al., 2015a; Rocha et al., 2016). nomen dubium according to Morek et al. (2016); not valid according to Suzuki (2016).

Class Eutardigrada Richters, 1926

Order Hypsibioidea Guil, Jørgensen \& Kristensen, 2019 Family Hypsibiidae Pilato, 1969

Adropion scoticum scoticum (Murray, 1905) (Barros, 1943; du Bois-Reymond Marcus, 1944; Mclnnes, 1994; Assunção, 1999b; Meyer, 2013; Kaczmarek et al., 2015a; Rocha et al., 2016). Subgenus Adropion of the genus Diphascon raised to genus level by Bertolani et al. (2014).

Diphascon alpinum Murray, 1906 (Rahm, 1931, 1932; Marcus, 1936, 1939; Mclnnes, 1994; Assunção, 1999b; Meyer, 2013; Kaczmarek et al., 2015a; Rocha et al., 2016).

Diphascon pingue pingue (Marcus, 1936) (du BoisReymond Marcus, 1944; Ramazzotti \& Maucci, 1983; Mclnnes, 1994; Assunção, 1999b; Meyer, 2013; Kaczmarek et al., 2015a; Rocha et al., 2016).

Hypsibius convergens (Urbanowicz, 1925) (Barros, 1943; du Bois-Reymond Marcus, 1944; Mclnnes, 1994; Assunção, 1999b; Meyer, 2013; Kaczmarek et al., 2015a; Rocha et al., 2016).

Hypsibius microps Thulin, 1928 (Barros, 1943; Ramazzotti \& Maucci, 1983; Mclnnes, 1994; Assunção, 1999b; Meyer, 2013; Kaczmarek et al., 2015a; Rocha et al., 2016).

Itaquascon umbellinae Barros, 1939 (Barros, 1939a; Ramazzotti \& Maucci, 1983; Kaczmarek et al., 2015a). Found in mosses, in Itaquaquecetuba and sampled by Berta and Ruth Lange de Morretes.

\section{Family Ramazzottiidae Sands, McInnes, Marley, Goodall-Copestake, Convey \& Linse, 2008}

Ramazzottius oberhaeuseri (Doyère, 1840) (Rahm, 1931, 1932; Marcus, 1936; Barros, 1943; du Bois-Reymond Marcus, 1944; Mclnnes, 1994; Assunção, 1999b; Meyer, 2013; Kaczmarek et al., 2015a; Rocha et al., 2016).

Order Isohypsibioidea Guil, Jørgensen \& Kristensen, 2019 Family Doryphoribiidae Gąsiorek, Stec, Morek \& Michalczyk, 2019

Doryphoribius evelinae (Marcus, 1928) (Marcus, 1936, 1939; Barros, 1943; du Bois-Reymond Marcus, 1944; Ramazzotti \& Maucci, 1983; Mclnnes, 1994; Assunção, 1999b; Meyer, 2013; Kaczmarek et al., 2015a; Rocha et al., 2016).

Doryphoribius flavus (Iharos, 1966) (Rocha et al., 2016).

Grevenius granulifer (Thulin, 1928) (du Bois-Reymond Marcus, 1944; Ramazzotti \& Maucci, 1983; Mclnnes,
1994; Assunção, 1999b; Meyer, 2013; Kaczmarek et al., 2015a; Rocha et al., 2016). Transferred from Isohypsibius by Gąsiorek et al. (2019c).

Grevenius myrops (du Bois-Reymond Marcus, 1944) (du Bois-Reymond Marcus, 1944; Ramazzotti \& Maucci, 1983; Mclnnes, 1994; Assunção, 1999b; Meyer, 2013; Kaczmarek et al., 2015a; Rocha et al., 2016). Found in the state of São Paulo, in aquariums of the Department of Zoology - USP, and in a stream in Santo Amaro; and in the state of Paraná, Curitiba, among aquatic plants. Transferred from Isohypsibius by Gąsiorek et al. (2019c). Pseudobiotus megalonyx (Thulin, 1928) (du Bois-Reymond Marcus, 1944; Kaczmarek et al., 2015a).

Thulinius augusti (Murray, 1907) (du Bois-Reymond Marcus, 1944; Mclnnes, 1994; Assunção, 1999b; Meyer, 2013; Rocha et al., 2016). Transferred from Isohypsibius by Bertolani (2003).

\section{Family Isohypsibiidae Sands, Mclnnes, Marley, Goodall-Copestake, Convey \& Linse, 2008}

Dianea papillifera (Murray, 1905) (Barros, 1943; du BoisReymond Marcus, 1944; Ramazzotti \& Maucci, 1983; Mclnnes, 1994; Assunção, 1999b; Meyer, 2013; Kaczmarek et al., 2015a; Rocha et al., 2016). Transferred from Isohypsibius by Gąsiorek et al. (2019c).

Fractonotus verrucosus (Richters, 1900) (du Bois-Reymond Marcus, 1944; Ramazzotti \& Maucci, 1983; Mclnnes, 1994; Assunção, 1999b; Meyer, 2013; Kaczmarek et al., 2015a; Rocha et al., 2016). Transferred from Calohypsibius by Gąsiorek et al. (2019a).

Isohypsibius sabellai Pilato, Binda, Napolitano \& Moncada, 2004 (Pilato et al., 2004; Meyer, 2013; Kaczmarek et al., 2015a). Found in Iguazu falls, state of Paraná, in a moss sample.

Ursulinius nodosus (Murray, 1907) (Marcus, 1936, 1939; Barros, 1943; du Bois-Reymond Marcus, 1944; Mclnnes, 1994; Assunção, 1999b; Meyer, 2013; Kaczmarek et al., 2015a; Rocha et al., 2016). Transferred from Isohypsibius by Gąsiorek et al. (2019c).

Order Macrobiotoidea Guil, Jørgensen \& Kristensen, 2019 Family Macrobiotidae Thulin, 1928

Macrobiotus echinogenitus Richters, 1903 (Rahm, 1931, 1932; Marcus, 1936, 1939; Mclnnes, 1994; Assunção, 1999b; Meyer, 2013; Kaczmarek et al., 2015a; Rocha et al., 2016).

Macrobiotus evelinae Barros, 1938 (Barros, 1938; Marcus, 1939; Ramazzotti \& Maucci, 1983; Mclnnes, 1994; Kaczmarek et al., 2015a; Rocha et al., 2016). Found in Capivarí, Campos de Jordão, in mosses on trees collected by Juanita Fortlage.

Macrobiotus furcatus Ehrenberg, 1859 (Barros, 1942b; du Bois-Reymond Marcus, 1944; Ramazzotti \& Maucci, 1983; Assunção, 1999b; Meyer, 2013; Kaczmarek et al., 2015a; Rocha et al., 2016). Re-transferred from Minibiotus by Bertolani et al. (2014). 
Macrobiotus hibiscus Barros, 1942 (Barros, 1942b; Ramazzotti \& Maucci, 1983; McInnes, 1994; Assunção, 1999b; Meyer, 2013; Kaczmarek et al., 2015a; Rocha et al., 2016). Found in Casa Branca, countryside of São Paulo.

Macrobiotus hufelandi Schultze, 1834 (Rahm, 1931, 1932; Marcus, 1939; Barros, 1942b; du Bois-Reymond Marcus, 1944; Mclnnes, 1994; Assunção, 1999b; Pulschen \& Meneghin, 2010; Meyer, 2013; Kaczmarek et al., 2015a; Rocha et al., 2016).

Macrobiotus occidentalis occidentalis Murray, 1910 (Murray, 1913; Marcus, 1936, 1939; du Bois-Reymond Marcus, 1944; Mclnnes, 1994; Meyer, 2013; Kaczmarek et al., 2015a; Rocha et al., 2016).

Macrobiotus polyopus Marcus, 1928 (Barros, 1942b; Ramazzotti \& Maucci, 1983; Mclnnes, 1994; Assunção, 1999b; Meyer, 2013; Kaczmarek et al., 2015a; Rocha et al., 2016).

Macrobiotus primitivae Barros, 1942 (Barros, 1942b; Ramazzotti \& Maucci, 1983; Mclnnes, 1994; Assunção, 1999b; Meyer, 2013; Kaczmarek et al., 2015a; Rocha et al., 2016). Found in Eldorado, state of São Paulo.

Macrobiotus psephus du Bois-Reymond Marcus, 1944 (du Bois-Reymond Marcus, 1944; Ramazzotti \& Maucci, 1983; Mclnnes, 1994; Assunção, 1999b; Meyer, 2013; Kaczmarek et al., 2015a; Rocha et al., 2016).

Macrobiotus sawayai Marcus, 1937 (Marcus, 1937; Assunção, 1999b; Kaczmarek et al., 2015a). Found in Pacaembu, state of São Paulo, in mosses on trees.

Mesobiotus coronatus (Barros, 1942) (Barros, 1942b; Mclnnes, 1994; Assunção, 1999b; Pilato et al., 2004; Meyer, 2013; Kaczmarek et al., 2015a; Rocha et al., 2016). Found in the city of Itapetininga. Transferred from Macrobiotus by Vecchi et al. (2016).

Mesobiotus furciger (Murray, 1907) (Rocha et al., 2016). Transferred from Macrobiotus by Vecchi et al. (2016).

Mesobiotus harmsworthi (Murray, 1907) (Marcus, 1939; du Bois-Reymond Marcus, 1944; Ramazzotti \& Maucci, 1983; Mclnnes, 1994; Assunção, 1999b; Meyer, 2013; Kaczmarek et al., 2015a; Rocha et al., 2016). Transferred from Macrobiotus by Vecchi et al. (2016).

Mesobiotus orcadensis (Murray, 1907) (Barros, 1942b; Ramazzotti \& Maucci, 1983; Mclnnes, 1994; Assunção, 1999b; Meyer, 2013; Kaczmarek et al., 2015a; Rocha et al., 2016). Transferred from Macrobiotus by Vecchi et al. (2016).

Mesobiotus stellaris (du Bois-Reymond Marcus, 1944) (du Bois-Reymond Marcus, 1944; Ramazzotti \& Maucci, 1983; Mclnnes, 1994; Assunção, 1999b; Meyer, 2013; Kaczmarek et al., 2015a; Rocha et al., 2016). Found in Campos do Jordão, state of São Paulo. Transferred from Macrobiotus by Vecchi et al. (2016).

Minibiotus acontistus (Barros, 1942) (Barros, 1942b; Ramazzotti \& Maucci, 1983; Mclnnes, 1994; Assunção, 1999b; Meyer, 2013; Kaczmarek et al., 2015a). Found in the city of Serra Negra. Transferred from Macrobiotus by Guidetti et al. (2007).

Minibiotus aculeatus (Murray, 1910) (Rocha et al., 2016).

Minibiotus intermedius (Plate, 1888) (Barros, 1942b; du Bois-Reymond Marcus, 1944; Mclnnes, 1994;
Assunção, 1999b; Meyer, 2013; Kaczmarek et al., 2015a; Rocha et al., 2016).

Minibiotus julietae (Barros, 1942) (Barros, 1942b; Ramazzotti \& Maucci, 1983; Mclnnes, 1994; Assunção, 1999b; Meyer, 2013; Kaczmarek et al., 2015a; Rocha et al., 2016). Found in Juqueri and Osasco cities, state of São Paulo. Transferred from Macrobiotus by Guidetti et al. (2007).

Minibiotus marcusi (Barros, 1942) (Barros, 1942b; Ramazzotti \& Maucci, 1983; Mclnnes, 1994; Assunção, 1999b; Meyer, 2013; Kaczmarek et al., 2015a; Rocha et al., 2016). Found near Santo Amaro, state of São Paulo. Transferred from Macrobiotus by Guidetti et al. (2007).

Paramacrobiotus (Amicrobiotus) cf. areolatus (Murray, 1907). Unpublished. Collected by the author in mosses from Curitiba, Paraná. Transferred from Macrobiotus by Guidetti et al. (2009).

Paramacrobiotus (Amicrobiotus) centesimus (Pilato, 2000) (Pilato, 2000; Meyer, 2013; Kaczmarek et al., 2015a). Found in Iguazu falls, state of Paraná, in a moss sample. Transferred from Macrobiotus by Guidetti et al. (2009).

Paramacrobiotus (Paramacrobiotus) richtersi (Murray, 1911) (Marcus, 1939; Barros, 1942b; du Bois-Reymond Marcus, 1944; Iharos, 1969; Kaczmarek et al., 2015a). Transferred from Macrobiotus by Guidetti et al. (2019).

Family Murrayidae Guidetti, Rebecchi \& Bertolani, 2000

Dactylobiotus ambiguus (Murray, 1907) (Rahm, 1931, 1932; Mclnnes, 1994; Assunção, 1999b; Meyer, 2013; Kaczmarek et al., 2015a; Rocha et al., 2016).

Murrayon pullari (Murray, 1907) (du Bois-Reymond Marcus, 1944; Mclnnes, 1994; Assunção, 1999b; Kaczmarek et al., 2015a; Rocha et al., 2016).

\section{Class Heterotardigrada Marcus, 1927 \\ Order Arthrotardigrada Marcus, 1927 \\ Family Archechiniscidae Binda, 1978}

Archechiniscus marci Schulz, 1953 (Rocha et al., 2013; Kaczmarek et al., 2015b; Miller \& Perry, 2016).

\section{Family Batillipedidae Ramazzotti, 1962}

Batillipes annulatus Zio, 1962 (Rocha et al., 2009, 2013; Kaczmarek et al., 2015b; Miller \& Perry, 2016).

Batillipes brasiliensis Santos, Rocha, Gomes Jr. \& Fontoura, 2017 (Santos et al., 2017). Found in shallow sublittoral medium to coarse, gravels and quartz sands, in low energy (Sossego Beach) and estuarine beaches (Forte Orange Beach and Gunga Beach), and in reef pools of high energy beaches (Amor Beach).

Batillipes dandarae Santos, Rocha, Gomes Jr. \& Fontoura, 2017 (Santos et al., 2017). Mainly found in shallow sublittoral fine to medium calcareous and 
quartz sands in low energy and estuarine beaches as Gunga Beach and Forte Orange Beach and occasionally in high energy beaches (Ponta do Sal) and in reef pools as in Patacho Beach.

Batillipes dicrocercus Pollock, 1970 (Rocha et al., 2009, 2013; Miller \& Perry, 2016).

Batillipes lesteri Kristensen \& Mackness, 2000 (Rocha et al., 2013; Kaczmarek et al., 2015b; Miller \& Perry, 2016).

Batillipes mirus Richters, 1909 (Höfling-Epiphanio, 1972; Assunção, 1999a; Rocha et al., 2013; Kaczmarek et al., 2015b; Miller \& Perry, 2016).

Batillipes pennaki Marcus, 1946 (Marcus, 1946; Ramazzotti \& Maucci, 1983; Assunção, 1999a; VictorCastro et al., 1999; Rocha et al., 2000, 2004, 2013; Kaczmarek et al., 2015b; Miller \& Perry, 2016; Santos et al., 2017). Found in sand and sea water from Ipanema Beach, state of Rio de Janeiro. Collected by Tagea Kristina S. Björnberg in 1946.

Batillipes potiguarensis Santos, Rocha, Gomes Jr. \& Fontoura, 2017 (Santos et al., 2017; Santos et al., 2018a). Found in shallow sublittoral medium to coarse gravels and quartz sands, in reef pools of high energy beaches (Amor Beach) and low energy beaches (Francês Beach).

\section{Family Halechiniscidae Thulin, 1928}

Chrysoarctus briandi Renaud-Mornant, 1984 (RenaudMornant, 1984; Rocha et al., 2013; Kaczmarek et al., 2015b; Miller \& Perry, 2016).

Dipodarctus subterraneus (Renaud-Debyser, 1959) (Rocha et al., 2009, 2013; Kaczmarek et al., 2015b; Miller \& Perry, 2016).

Florarctus hulingsi Renaud-Mornant, 1976 (Rocha et al., 2013; Kaczmarek et al., 2015b; Miller \& Perry, 2016).

Halechiniscus perfectus Schulz, 1955 (Moura et al., 2009; Rocha et al., 2013; Kaczmarek et al., 2015b; Miller \& Perry, 2016).

Halechiniscus tuleari Renaud-Mornant, 1979 (Rocha et al., 2013; Kaczmarek et al., 2015b; Miller \& Perry, 2016).

Ligiarctus alatus Gomes Júnior, Santos, da Rocha, Santos \& Fontoura, 2018 (Gomes Júnior et al., 2018). Found in Potiguar Basin, state of Rio Grande do Norte, at $150 \mathrm{~m}$ below the sea level in bioclastic and litoclastic sand.

Opydorscus fonsecae Renaud-Mornant, 1989 (RenaudMornant, 1989; Assunção, 1999a; Rocha et al., 2013; Kaczmarek et al., 2015b; Miller \& Perry, 2016). Found in continental shelf of Fortaleza, state of Ceará, depth $40 \mathrm{~m}$, in fine sediments, sandy-muddy.

Orzeliscus belopus du Bois Raymond-Marcus, 1952 (du Bois-Reymond Marcus, 1952; Ramazzotti \& Maucci, 1983; Assunção, 1999a; Rocha et al., 2013; Kaczmarek et al., 2015b; Miller \& Perry, 2016; Santos et al., 2017). Found in the coast of Island of São Sebastião, near Ilhabela, state of São Paulo, in 3-5 depth, sand with rather much detritus.

Wingstrandarctus intermedius (Renaud-Mornant, 1967) (Rocha et al., 2009, 2013; Kaczmarek et al., 2015b; Miller \& Perry, 2016).
Family Neoarctidae (Grimaldi de Zio, D’Addabbo Gallo \& Morone De Lucia, 1992)

Neoarctus sp. (Rocha et al., 2013; Miller \& Perry, 2016).

\section{Family Neostygarctidae de Zio Grimaldi, D’Addabbo Gallo \& De Lucia Morone, 1987}

Neostygarctus sp. (Rocha et al., 2013; Miller \& Perry, 2016).

\section{Family Stygarctidae Schulz, 1951}

Mesostygarctus intermedius (Renaud-Mornant, 1979) (Rocha et al., 2009, 2013; Kaczmareket al., 2015b; Miller \& Perry, 2016). Transferred from Pseudostygarctus by Hansen et al. (2012).

Parastygarctus sterreri Renaud-Mornant, 1970 (Moura et al., 2009; Rocha et al., 2013; Kaczmarek et al., 2015b; Miller \& Perry, 2016).

Stygarctus bradypus Schulz, 1951 (Rocha et al., 2009, 2013; Verçosa et al., 2009; Kaczmarek et al., 2015b; Miller \& Perry, 2016).

\section{Family Styraconyxidae Kristensen \& Renaud-Mornant, 1983}

Angursa lingua Bussau, 1992 (Rocha et al., 2013; Kaczmarek et al., 2015b; Miller \& Perry, 2016).

Raiarctus aureolatus Renaud-Mornant, 1981 (Rocha et al., 2013; Kaczmarek et al., 2015b).

\section{Family Tanarctidae Renaud-Mornant, 1980}

Actinarctus doryphorus doryphorus Schulz, 1935 (Rocha et al., 2013; Kaczmarek et al., 2015b; Miller \& Perry, 2016).

Tanarctus dendriticus Renaud-Mornant, 1980 (RenaudMornant, 1980; Rocha et al., 2013; Kaczmarek et al., 2015b; Miller \& Perry, 2016).

Tanarctus heterodactylus Renaud-Mornant, 1980 (Renaud-Mornant, 1980; Rocha et al., 2013; Kaczmarek et al., 2015b; Miller \& Perry, 2016).

Tanarctus velatus McKirdy, Schmidt \& McGinty-Bayly, 1976 (Rocha et al., 2013; Kaczmarek et al., 2015b; Miller \& Perry, 2016).

\section{Order Echiniscoidea Richters, 1926 \\ Family Echiniscidae Thulin, 1928}

Bryochoerus intermedius intermedius Murray, 1910 (Barros, 1942a; Ramazzotti \& Maucci, 1983; Mclnnes, 1994; Assunção, 1999b; Meyer, 2013; Kaczmarek et al., 2015a; Rocha et al., 2016).

Bryodelphax alzirae (du Bois-Reymond Marcus, 1944) (du Bois-Reymond Marcus, 1944; Ramazzotti \& Maucci, 
1983; Mclnnes, 1994; Assunção, 1999b; Meyer, 2013; Kaczmarek et al., 2015a; Rocha et al., 2016). Found in the littoral zone of Bertioga, state of São Paulo.

Bryodelphax parvulus Thulin, 1928 (Barros, 1942a; Ramazzotti \& Maucci, 1983; Mclnnes, 1994; Assunção, 1999b; Meyer, 2013; Kaczmarek et al., 2015a; Rocha et al., 2016).

Echiniscus arctomys Ehrenberg, 1853 (Rahm, 1931, 1932; Marcus, 1936, 1939; Mclnnes, 1994; Meyer, 2013; Kaczmarek et al., 2015a). Nomen inquirendum according to Gąsiorek et al. (2019b).

Echiniscus blumi blumi Richters, 1903 (Rahm, 1931, 1932; Marcus, 1936, 1939; Barros, 1942a; Mclnnes, 1994; Assunção, 1999b; Meyer, 2013; Kaczmarek et al., 2015a; Rocha et al., 2016).

Echiniscus crassispinosus crassispinosus Murray, 1907 (Barros, 1942a; du Bois-Reymond Marcus, 1944; Assunção, 1999b; Meyer, 2013; Kaczmarek et al., 2015a).

Echiniscus crassispinosus fasciatus Marcus, 1928 (Barros, 1942a; du Bois-Reymond Marcus, 1944; Ramazzotti \& Maucci, 1983; Mclnnes, 1994; Assunção, 1999b; Meyer, 2013; Kaczmarek et al., 2015a; Rocha et al., 2016).

Echiniscus dreyfusi Barros, 1942 (Barros, 1942a; Ramazzotti \& Maucci, 1983; Mclnnes, 1994; Assunção, 1999b; Meyer, 2013; Kaczmarek et al., 2015a; Rocha et al., 2016). Found in São Vicente, state of São Paulo.

Echiniscus duboisi Richters, 1902 (Barros, 1942a; Ramazzotti \& Maucci, 1983; Mclnnes, 1994; Assunção, 1999b; Meyer, 2013; Kaczmarek et al., 2015a; Rocha et al., 2016).

Echiniscus evelinae Barros, 1942 (Barros, 1942a; du Bois-Reymond Marcus, 1944; Ramazzotti \& Maucci, 1983; Mclnnes, 1994; Assunção, 1999b; Meyer, 2013; Kaczmarek et al., 2015a; Rocha et al., 2016). Found in Barretos, state of São Paulo.

Echiniscus spiniger Richters, 1904 (Barros, 1942a; du BoisReymond Marcus, 1944; Mclnnes, 1994; Assunção, 1999b; Kaczmarek et al., 2015a; Rocha et al., 2016).

Echiniscus tenuis Marcus, 1928 (du Bois-Reymond Marcus, 1944; Ramazzotti \& Maucci, 1983; Mclnnes, 1994; Assunção, 1999b; Meyer, 2013; Kaczmarek et al., 2015a; Rocha et al., 2016).

Echiniscus testudo (Doyère, 1840) (Rahm, 1931, 1932; Marcus, 1936, 1939; Barros, 1942a; Mclnnes, 1994; Assunção, 1999b; Meyer, 2013; Kaczmarek et al., 2015a; Rocha et al., 2016).

Mopsechiniscus imberbis (Richters, 1907) (du BoisReymond Marcus, 1944; Ramazzotti \& Maucci, 1983; McInnes, 1994; Rocha et al., 2016).

Mopsechiniscus schusteri Dastych, 1999 (du BoisReymond Marcus, 1944; Assunção, 1999b; Dastych, 2000; Meyer, 2013).

Nebularmis phocae (du Bois-Reymond Marcus, 1944) (du Bois-Reymond Marcus, 1944; Ramazzotti \&Maucci, 1983; Mclnnes, 1994; Assunção, 1999b; Meyer, 2013; Kaczmarek et al., 2015a; Rocha et al., 2016). Found in Campos do Jordão, state of São Paulo, nearly 1.900 m. Transferred from Echiniscus by Gąsiorek et al. (2019b).
Pseudechiniscus bispinosus (Murray, 1907) (Rahm, 1931, 1932; Marcus, 1936, 1939; Ramazzotti \& Maucci, 1983; McInnes, 1994; Meyer, 2013; Kaczmarek et al., 2015a; Rocha et al., 2016).

Pseudechiniscus facettalis Petersen, 1951 (Mclnnes, 1994; Meyer, 2013).

Pseudechiniscus juanitae Barros, 1939 (Barros, 1939b, 1942a; du Bois-Reymond Marcus, 1944; Ramazzotti \& Maucci, 1983; Mclnnes, 1994; Assunção, 1999b; Meyer, 2013; Kaczmarek et al., 2015a; Rocha et al., 2016). Found in Campos do Jordão, state of São Paulo, collected by Juanita Fortlage.

Pseudechiniscus novaezeelandiae novaezeelandiae (Richters, 1908) (Barros, 1942a; du Bois-Reymond Marcus, 1944; Mclnnes, 1994; Assunção, 1999b; Meyer, 2013; Kaczmarek et al., 2015a).

Pseudechiniscus novaezeelandiae aspinosa Iharos, 1963 (Rocha et al., 2016).

Pseudechiniscus novaezeelandiae marinae Bartoš, 1934 (Marcus, 1939; Ramazzotti \& Maucci, 1983; Mclnnes, 1994; Kaczmarek et al., 2015a; Rocha et al., 2016).

Pseudechiniscus suillus (Ehrenberg, 1853) (Rahm, 1931, 1932; Marcus, 1936, 1939; Barros, 1942a; du BoisReymond Marcus, 1944; Mclnnes, 1994; Assunção, 1999b; Meyer, 2013; Kaczmarek et al., 2015a; Rocha et al., 2016).

Testechiniscus macronyx (Richters, 1907) (Barros, 1942a; Assunção, 1999b; Meyer, 2013; Kaczmarek et al., 2015a; Rocha et al., 2016).

Viridiscus rufoviridis (du Bois-Reymond Marcus, 1944) (du Bois-Reymond Marcus, 1944; Ramazzotti \& Maucci, 1983; Mclnnes, 1994; Assunção, 1999b; Meyer, 2013; Kaczmarek et al., 2015a; Rocha et al., 2016). Found in Campos do Jordão, state of São Paulo, in mosses on trees. Transferred from Echiniscus by Gąsiorek et al. (2019b).

Viridiscus viridis (Murray, 1910) (Marcus, 1936, 1939; Barros, 1942a; du Bois-Reymond Marcus, 1944; Ramazzotti \& Maucci, 1983; Mclnnes, 1994; Assunção, 1999b; Meyer, 2013; Kaczmarek et al., 2015a; Rocha et al., 2016). Transferred from Echiniscus by Gąsiorek et al. (2019b).

\section{Family Echiniscoididae Kristensen \& Hallas, 1980}

Echiniscoides sigismundi sigismundi (M. Schultze, 1865) (du Bois-Reymond Marcus, 1952; Assunção, 1999a; Rocha et al., 2013; Kaczmarek et al., 2015b; Miller \& Perry, 2016).

\section{ACKNOWLEDGEMENTS}

The author is grateful to Łukasz Kaczmarek (Adam Mickiewicz University, Poland), and Gisela Aramiriam León Espinosa (Universidad Autónoma de Nuevo León, Mexico) for providing historic references which were essential to the realization of this paper. 


\section{REFERENCES}

Aguinaldo, A.M.; Turbeville, J.M.; Linford, L.S.; Rivera, M.C.; Garey, J.R.; Raff, R.A. \& Lake, J.A. 1997. Evidence for a clade of nematodes, arthropods and other moulting animals. Nature, 387: 489-493.

Assunção, C.M.L. 1994. Análise filogenética em Tardigrada. (Masters Dissertation). Universidade Federal da Paraíba, João Pessoa. 54p.

Assunção, C.M.L. 1999a. Tardigrada. In: Joly, C.A. \& Bicudo, C.E.M. Biodiversidade do Estado de São Paulo: Síntese do Conhecimento ao Final do Século XX - Invertebrados Marinhos. São Paulo, Fundação de Amparo à Pesquisa do Estado de São Paulo. v. 3, p. 185-189.

Assunção, C.M.L. 1999b. Tardigrada. In: Ismael, D.; Valenti, W.C. \& MatsumuraTundisi, T. Biodiversidade do Estado do São Paulo: Síntese do Conhecimento ao Final do Século XX - Invertebrados de Água Doce. São Paulo, Fundação de Amparo à Pesquisa do Estado de São Paulo. v. 4, p. 61-64.

Assunção, C.M.L. 2001. Análise filogenética em Macrocephala (Tardigrada, Archeotardigrada). (Doctoral Thesis). Universidade de São Paulo, São Paul0. 87p.

Barros, R. 1938. Macrobiotus evelinae, uma nova especie de tardígrados. Boletim Biológico (Nova Série), 3: 52-54.

Barros, R. 1939a. Itaquascon umbellinae gen. nov. sp. nov. (Tardigrada, Macrobiotidae). Zoologischer Anzeiger, 128: 106-109.

Barros, R. 1939b. Pseudobiotus juanitae nova especie de tardígrado. Boletim Biológico (Nova Série), 4: 367-368.

Barros, R. 1942a. Tardígrados do estado de São Paulo, Brasil. I. Introdução. Gêneros "Echiniscus" e "Pseudechiniscus". Revista Brasileira de Biologia, 2: 257-269.

Barros, R. 1942b. Tardígrados do estado de São Paulo, Brasil. II. Gênero Macrobiotus. Revista Brasileira de Biologia, 2: 373-386.

Barros, R. 1943. Tardígrados do estado de São Paulo, Brasil. III. Gêneros Hypsibius, Itaquascon e Milnesium. Revista Brasileira de Biologia, 3: 1-10.

Bertolani, R. 2003. Thulinius, new generic name substituting for Thulinia Bertolani, 1981 (Tardigrada, Eutardigrada). Zootaxa, 314: 1-4.

Bertolani, R.; Guidetti, R.; Marchioro, T.; Altiero, T.; Rebecchi, L. \& Cesari, M. 2014. Phylogeny of Eutardigrada: New molecular data and their morphological support lead to the identification of new evolutionary lineages. Molecular Phylogenetics and Evolution, 76: 110-126.

Corrêa, D.C. 1987. Tardigrada. In: Sociedade Brasileira de Zoologia. Manual de técnicas para preparações zoológicas. Campinas, Sociedade Brasileira de Zoologia. v. 15, p. 1-6.

Corrêa, D.D. 1991. Dr. Eveline du Bois-Reymond Marcus. Hydrobiologia, 227: 23-26.

Dastych, H. 2000. Redescription of the Neotropical tardigrade Mopsechiniscus granulatus Mihelcic, 1967 (Tardigrada). Mitteilungen Hamburgisches Zoologisches Museum und Institut, 97: 45-57.

Degma, P.; Bertolani, R. \& Guidetti, R. 2019. Actual checklist of Tardigrada species (35 th Edition, 31-07-2019). Available at: http://www.evozoo. unimore.it/site/home/tardigrade-tools/documento1080026927.html. Access in: 21/09/2019.

Du Bois-Reymond Marcus, E. 1944. Sobre Tardígrados Brasileiros. Comunicaciones Zoologicas del Museo de Historia Natural de Montevideo, 13: 1-28.

Du Bois-Reymond Marcus, E. 1952. On South American Malacopoda. Boletins da Faculdade de Philosophia, Sciencias e Letras, Universidade de São Paulo -Zoologia, 17: 189-209.

Edmunds, M. 1991. Eveline du Bois-Reymond Marcus. Journal of Molluscan Studies, 57: 141-142.

Formiga, D.0. 2010. A escola tropical de genética brasileira. Ciência \& Cultura, 62(2): 12-13.

Garraffoni, A.R.S. 2019. Currículo Lattes. Available at: http://lattes.cnpq. br/1003535723082551. Access in: 23/09/2019.
Gąsiorek, P.; Morek, W.; Stec, D.; Blagden, B. \& Michalczyk, Ł. 2019a. Revisiting Calohypsibiidae and Microhypsibiidae: Fractonotus Pilato, 1998 and its phylogenetic position within Isohypsibiidae (Eutardigrada: Parachela). Zoosystema, 41(6): 71-89.

Gąsiorek, P.; Morek, W.; Stec, D. \& Michalczyk, Ł. 2019b. Untangling the Echiniscus Gordian knot: paraphyly of the "arctomys group" (Heterotardigrada: Echiniscidae). Cladistics, 1-21.

Gąsiorek, P.; Stec, D.;Morek, W. \& Michalczyk, Ł. 2019c. Deceptive conservatism of claws: distinct phyletic lineages concealed within Isohypsibioidea (Eutardigrada) revealed by molecular and morphological evidence. Contributions to Zoology, 88: 78-132.

Gomes Júnior, E.L. 2015. Tardigrada. In: Boeger, W.A.; Zaher, H.; Rafael, J.A. \& Valim, M.P. Catálogo Taxonômico da Fauna do Brasil. Available at: http:// fauna.jbri.gov.br/fauna/faunadobrasil/21. Access in: 09/12/2018.

Gomes Júnior, E.L. \& Rocha, C.M.C. 2015. Microscopia eletrônica de varredura com tardígrados (Filo Tardigrada): sugestão de protocolos adaptados às peculiaridades do grupo. Revista Nordestina de Zoologia, 9(2): 16-32.

Gomes Júnior, E.L.; Santos, E.; Rocha, C.M.C.; Santos, P.J.P. \& Fontoura, P. 2018. A new species of Ligiarctus (Tardigrada, Arthrotardigrada) from the Brazilian continental shelf, Southwestern Atlantic Ocean. Marine Biodiversity, 48(1): 5-12.

Grothman, G.T.; Johansson, C. \& Chilton, G. 2017. Gilbert Rahm and the status of Mesotardigrada Rahm, 1937. Zoological Science, 34(1): 5-10.

Guidetti, R.; Bertolani, R. \& Degma, P. 2007. New taxonomic position of several Macrobiotus species (Eutardigrada: Macrobiotidae). Zootaxa, 1471: 61-68

Guidetti, R.; Schill, R.0.; Bertolani, R.; Dandekar, T. \& Wolf, M. 2009. New molecular data for tardigrade phylogeny, with the erection of Paramacrobiotus gen. n. Journal of Zoological Systematics and Evolutionary Research, 47(4): 315-321.

Guidetti, R.; Cesari, M.; Bertolani, R.; Altiero, T. \& Rebecchi, L. 2019. High diversity in species, reproductive modes and distribution within the Paramacrobiotus richtersi complex (Eutardigrada, Macrobiotidae). Zoological Letters, 5(1): 1-28.

Guil, N.; Jørgensen, A. \& Kristensen, R. 2019. An upgraded comprehensive multilocus phylogeny of the Tardigrada tree of life. Zoologica Scripta, 48(1): 120-137.

Hansen, J.G.; Kristensen, R.M. \& Jørgensen, A. 2012. The armoured marine tardigrades (Arthrotardigrada, Tardigrada). Scientia Danica, Series B, Biologica, 2: 1-91.

Höfling-Epiphanio, E. 1972. Ocorrência de Batillipes mirus Richters, 1909 e B. tubernatis Pollock, 1971 (Tardigrada) no litoral brasileiro. Ciência \& Cultura, 24:358-359.

Iharos, G. 1969. The Scientific Results of the Hungarian Soil Zoological Expeditions to South America. Öpuscula Zoologica Budapest, 9(2): 279-289.

Jørgensen, A.; Kristensen, R.M. \& Mobjerg, N. 2018. Phylogeny and Integrative Taxonomy of Tardigrada. In: Schill, R.O. (Ed.). Water Bears: The Biology of Tardigrades. Switzerland, Springer International Publishing. p. 95-114. (Zoological Monographs 2).

Kaczmarek, Ł.; Michalczyk, Ł. \& McInnes, S.J. 2015a. Annotated zoogeography of non-marine Tardigrada. Part Il: South America. Zootaxa, 3923: 1-107.

Kaczmarek, Ł.; Bartels, P.J.; Roszkowska, M. \& Nelson, D.R. 2015b. The Zoogeography of marine Tardigrada. Zootaxa, 4037: 1-189.

Marcus, E. 1936. Tardigrada. Das Tierreich, 66: 1-340.

Marcus, E. 1937. Sôbre a anabiose dos Tardigrados, com descrição de uma nova espécie. Boletim Biologico, 3(5): 7-13.

Marcus, E. 1939. IV. Tardigrada. The Percy Sladen Trust Expedition to Lake Titicaca. Transactions of the Linnean Society of London, 3: 45-49. 
Marcus, E. 1946. Batillipes pennaki, a new marine tardigrade from the North and South american Atlantic coast. Comunicaciones Zoologicas del Museo de Historia Natural de Montevideo, 33(2): 1-3.

McInnes, S.J. 1994. Zoogeographic distribution of terrestrial/freshwater tardígrados from current literature. Journal of Natural History, 28: 257-352.

Mendes, E.G. 1994. Ernst Marcus. Estudos Avançados, 8(22): 209-213.

Meyer, H.A. 2013. Terrestrial and freshwater Tardigrada of the Americas. Zootaxa, 3747: 1-71.

Miller, W.R. \& Perry, E.S. 2016. The coastal marine Tardigrada of the Americas. Zootaxa, 4126(3): 375-396.

Morek, W.; Gąsiorek, P.; Stec, D.; Blagden, B. \& Michalczyk, Ł. 2016. Experimental taxonomy exposes ontogenetic variability and elucidates the taxonomic value of claw configuration in Milnesium Doyère, 1840 (Tardigrada: Eutardigrada: Apochela). Contributions to Zoology, 85: 173-200.

Moura, J.R.; Verçosa, M.M.; Santos, E.C.L.; Santana e Silva, L.G.; Amaral, F.M.D. \& Rocha, C.M.C. 2009. Ocorrência de Parastygarctus sterreri RenaudMornant, 1970 e Halechiniscus perfectus Schulz, 1955 (Tardigrada, Heterotardigrada) no Arquipélago de São Pedro e São Paulo, RN, Brasil. Atlântica, Rio Grande, 31(2): 213-216.

Murray, J. 1913. Notes on the natural history of Bolivia and Peru: including a report on the Rhizopoda / by G.H. Wailes. Scottish, Edinburgh Oceanographic Laboratory,.

Nelson, D.R. 2002. Current status of the Tardigrada: evolution and ecology. Integrative and Comparative Biology, 42: 652-659.

Nelson, D.R.; Guidetti, R. \& Rebecchi, L. 2015. Phylum Tardigrada. In: Thorp, J. \& Rogers, D.C. Ecology and General Biology: Thorp and Covich's Freshwater Invertebrates. 4. ed. London,Academic Press, p. 347-380.

Pilato, G. 2000. Macrobiotus centesimus, new species of eutardigrade from the South America. Bollettino dell'Accademia Gioenia di Scienze Naturali di Catania, 33: 97-101.

Pilato, G.; Binda M.G.; Napolitano, A. \& Moncada, E. 2004. Remarks on some species of tardigrades from South America with the description of two new species. Journal of Natural History, 38: 1081-1086.

Pulschen, A.A. \& Meneghin, S.P. 2010. Estabelecimento de uma cultura de tardígrados limno-terrestres em laboratório e desenvolvimento de metodologias alternativas de desidratação de tardígrados. Evidência, Joaçaba, 10(1-2): 69-85.

Rahm, G. 1931. Tardigrada of the South of America (esp. Chile). Revista Chilena de História Natural, 35: 118-141.

Rahm, G. 1932. Freilebende Nematoden, Rotatorien und Tardigraden aus Südamerika (besonders aus Chile). C. Tardigrada. Zoologischer Anzeiger, 98: 113-128.

Ramazzotti, G. \& Maucci, W. 1983. The Phylum Tardigrada. 3. ed., Verbania Pallanza. Instituto Italiano di Idrobiologia. 1012p.

Renaud-Mornant, J. 1980. Description de trois espèces nouvelles du genre Tanarctus Renaud-Debyser, 1959 et création de la sous-famille des tanarctinae, subfam. nov. (Tardigrada, Heterotardigrada). Bulletin du Muséum National d'Histoire Naturelle, 4: 129-141.

Renaud-Mornant, J. 1984. Halechiniscidae (Heterotardigrada) de la campagne Benthedi, canal du Mozambique. Bulletin du Muséum National d'Histoire Naturelle, 6: 67-88.

Renaud-Mornant, J. 1989. Opydorscus, un nouveau genre d'Orzeliscinae et sa signification phylogénique (Tardigrada, Arthrotardigrada). Bulletin du Muséum National d'Histoire Naturelle, Paris, 11(4): 763-771.
Rocha, C.M.C. 2018. Currículo Lattes. Available at: http://lattes.cnpq. br/8971495967943784. Access in: 25/10/2018.

Rocha, C.M.C.; Fonsêca-Genevois, V. \& Castro, F.J.V. 2000. Distribuição espaço-temporal de Batillipes pennaki Marcus, 1946 (Tardigrada, Heterotardigrada) na margem sul da llha de Itamaracá (Pernambuco Brasil). Tropical Oceanography, 28: 35-46.

Rocha, C.M.C.; Fonsêca-Genevois, V.; Victor-Castro, F.J.; Bezerra, T.N.C.; Venekey, V. \& Botelho, A.P. 2004. Environmental interactions of Batillipes pennaki (Tardigrada, Heterotardigrada) in a tropical sandy beach (Itamaracá, Pernambuco, Brazil). Meiofauna Marina, 13: 79-86.

Rocha, C.M.C.; Verçosa, M.M.; Santos, E.C.L.; Barbosa, D.F.; Oliveira, D.A.S. \& Souza, J.R.B. 2009. Marine tardigrades from the coast of Pernambuco, Brazil. Meiofauna Marina, 17: 97-101.

Rocha, C.M.C.; Santos, E.C.L.; Gomes Júnior, E.L.; Moura, J.R.; Santana e Silva, L.G. \& Barbosa, D.F. 2013. New records of marine tardigrades from Brazil. Journal of Limnology, 72(S1): 102-107.

Rocha, C.M.C.; Gomes Júnior, E.L. \& Santos, E.C.L. 2016. Brazilian limnoterrestrial tardigrades (Bilateria, Tardigrada): new occurrences and species checklist updates. Revista Nordestina de Zoologia, 10: 21-31.

Santos, E.; Rocha, C.M.C.; Gomes Jr., E. \& Fontoura, P. 2017. Three new Batillipes species (Arthrotardigrada: Batillipedidae) from the Brazilian coast. Zootaxa, 4243(3): 483-502.

Santos, E.; Gomes Jr., E. Rocha, C.M.C.; Bartels, P.J. \& Fontoura, P. 2018a. A closer look at Batillipes dicrocercus and new records of Batillipes potiguarensis (Tardigrada: Arthrotardigrada) from the Brazilian coast with comments on intra- and interspecific variability. Iheringia, Série Zoologia, 108: 1-10.

Santos, E.; Rubal, M.; Veiga, P.; Bartels, P.J.; Rocha, C.M.C. \& Fontoura, P. 2018b. On the distribution of Batillipes tubernatis Pollock, 1971 (Arthrotardigrada: Batillipedidae) in the Atlantic Basin. Marine Biodiversity, 1-11.

Sawaya, P. 1970. Professor Dr. Ernst Gotthelf Marcus. Boletim de Instituto de Biociências da Universidade de São Paulo, 1(27): 5-18.

Suzuki, A.C. 2016. Specimens with an artifact appearing as 'three spines' in Milnesium tardigradum var. trispinosa Rahm, 1931 (Tardigrada). Zoological Science, 33: 431-433.

Universidade de São Paulo (USP). 2019. Histórico do Departamento de Zoologia do IB-USP. Available at: https://www.ib.usp.br/dep-zoologia/historico. html. Access in: 23/09/2019.

Vecchi, M.; Cesari, M.; Bertolani, R.; Jönsson, K.I.; Rebecchi, L. \& Guidetti, R. 2016. Integrative systematic studies on tardigrades from Antarctica identify new genera and new species within Macrobiotoidea and Echiniscoidea. Invertebrate Systematics, 30: 303-322.

Verçosa, M.M.; Santos, E.C.L.; Souza, J.R.B. \& Rocha, C.M.C. 2009. Distribuição de Stygarctus bradypus Schulz, 1951 (Tardigrada, Heterotardigrada) na margem sul da llha de Itamaracá (PE, Brasil). Atlântica, Rio Grande, 31: 25-33.

Victor-Castro, F.J.; Fonsêca-Genevois, V.; Lira, L. \& Rocha, C.M.C. 1999. Efeito da sedimentação sobre a distribuição de Batillipes pennaki Marcus, 1946 em zona tropical típica: Restinga do Paiva, Pernambuco, Brazil. Tropical Oceanography, 27(2): 89-102. 